\title{
Laboratory simulation of the evolution of organic matter in dense interstellar clouds
}

\author{
Vito Mennella \\ Istituto Nazionale di Astrofisica, Osservatorio Astronomico di Capodimonte, \\ via Moiariello 16, 80131 Napoli, Italy \\ email: mennella@na.astro.it
}

\begin{abstract}
Laboratory simulation of interstellar grain processing is a unique tool to better understand the nature and evolution of cosmic dust. In recent years this approach has been crucial to outline a new model of evolution of the aliphatic component of organic matter in the interstellar medium. Here, the results of a recent laboratory research on processing of nanosized carbon particles by $\mathrm{H}$ atoms under simulated dense medium conditions are discussed. The experiments show that the formation of $\mathrm{C}-\mathrm{H}$ bonds in the aliphatic $\mathrm{CH}_{2}$ and $\mathrm{CH}_{3}$ functional groups does not take place, while the activation of a band at $3.47 \mu \mathrm{m}$, due to the $\mathrm{C}-\mathrm{H}$ stretching vibration of tertiary $s p^{3}$ carbon atoms, is observed. These results indicate that the assumption about inhibition of aliphatic C-H bond formation in interstellar dense clouds is correct. Moreover, they suggest that carbon grains responsible for the interstellar aliphatic band at $3.4 \mu \mathrm{m}$ in diffuse regions can contribute to the absorption observed at $3.47 \mu \mathrm{m}$ in dense clouds.
\end{abstract}

Keywords. ISM: dust, extinction, methods: laboratory

\section{Introduction}

The presence of an aliphatic organic dust component in diffuse regions of the interstellar medium is indicated by the $3.4 \mu \mathrm{m}$ absorption band with subfeatures at $3.38,3.42$, and $3.48 \mu \mathrm{m}$ due to the $\mathrm{C}-\mathrm{H}$ stretching modes in the methyl $\left(\mathrm{CH}_{3}\right)$ and methylene groups $\left(\mathrm{CH}_{2}\right)$ (Sandford et al. 1991, Pendleton et al. 1994). The carrier of the feature is a widespread component of diffuse interstellar dust. The $3.4 \mu \mathrm{m}$ band is absent in the spectrum of dense cloud dust: the number of aliphatic $\mathrm{C}-\mathrm{H}$ bonds is reduced by at least $55 \%$ with respect to the diffuse regions (Muñoz Caro et al. 2001). In dense clouds, the $\mathrm{C}-\mathrm{H}$ stretching spectral region is generally characterized by the absorption bands at 3.25, 3.54 and $3.47 \mu \mathrm{m}$. Allamandola et al. (1992) first detected the band at $3.47 \mu \mathrm{m}$ in four protostars. Subsequent observations have confirmed that the $3.47 \mu \mathrm{m}$ band is a common feature of young stellar objects embedded in molecular clouds (Sellgren et al. 1994, Brooke et al. 1996, 1999). Chiar et al. (1996) reported the first detection of the band in quiescent medium along the line of sight of the field star Elias 16 in the Taurus cloud. The optical depth of the $3.47 \mu \mathrm{m}$ band is better correlated with the optical depth of the $3 \mu \mathrm{m}$ water ice band than with that of the $9.7 \mu \mathrm{m}$ silicate absorption feature, suggesting that the absorption is not due to a refractory component (Chiar et al. 1996, Brooke et al. 1999). Allamandola et al. (1992) attributed the band to the C-H stretching vibration of tertiary $s p^{3}$ carbon atoms, and they suggested interstellar diamond grains as carrier of the feature. More recently, the band has been assigned to a vibrational mode due to the interaction of the nitrogen atom of $\mathrm{NH}_{3}$ with an $\mathrm{OH}$ bond of the water molecule, forming an ammonia hydrate (Dartois et al. 2002).

The distinct spectral difference between dense and diffuse medium represents a strong constraint for any description of formation and evolution of interstellar organic matter 
(Allamandola et al. 1993). It has long been enigmatic. In recent years, on the basis of specific laboratory simulation of interstellar processing, a model has been proposed to reconcile the dichotomy of the $3.4 \mu \mathrm{m}$ band through an evolutionary transformation of interstellar organic matter. During cycling of materials between diffuse and dense interstellar regions the degree of processing and the properties of grains change and these changes determine the observed spectral variations. In the diffuse medium, bare carbon grains are processed by UV photons and cosmic rays, which destroy $\mathrm{C}-\mathrm{H}$ bonds, and $\mathrm{H}$ atoms, which form aliphatic bonds. Competition between destruction and formation determines an equilibrium value for grain hydrogenation and we can observed the $3.4 \mu \mathrm{m}$ band. In dense regions an ice layer, consisting primarily of $\mathrm{H}_{2} \mathrm{O}$, covers grains. In these conditions destruction of $\mathrm{C}-\mathrm{H}$ bonds by cosmic rays and the internal UV field remains active, while their formation should stop, determining a gradual reduction of the intensity of the C-H stretching feature (Mennella et al. 1999, 2001, 2002, 2003, Muñoz Caro et al. 2001, Mennella 2006). The previous model relies on the results of specific laboratory studies of the interaction of UV photons, ions, and hydrogen atoms with hydrogen-free and hydrogenated carbon grains under simulated diffuse and dense medium conditions. From these experiments the $\mathrm{C}-\mathrm{H}$ bond formation and destruction cross sections have been obtained. Knowledge of these quantities has allowed a quantitative description of the evolution of the aliphatic component.

All processes driving the evolution of interstellar organic matter have been studied in the laboratory, except for the intercation of $\mathrm{H}$ atoms with carbon grains covered with an ice cap.

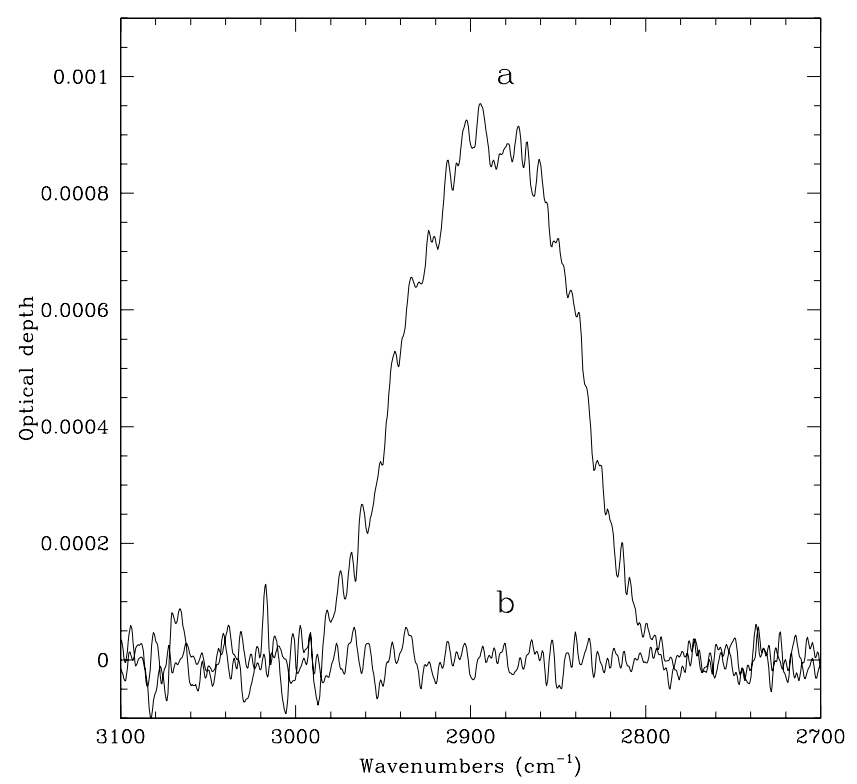

Figure 1. (a) Activation of the $2883 \mathrm{~cm}^{-1}(3.47 \mu \mathrm{m})$ band after $\mathrm{H}$ atom exposure to $1.0 \times 10^{18}$ atoms $\mathrm{cm}^{2}$ of nano sized carbon grains covered with a water ice layer at $12 \mathrm{~K}$.

(b) For comparison, the spectrum of a water ice layer, with a thickness of $27 \mathrm{~nm}$, deposited on a Cs I window after an irradiation of $1.4 \times 10^{18}$ atoms $\mathrm{cm}^{2}$.

\section{H atom irradiation of carbon grains in dense clouds}

Only recently have simulations of the evolution of organic matter in the ISM been completed with laboratory research on $\mathrm{H}$ atom processing of nano-sized carbon particles 
under simulated dense medium conditions. The variations of the degree of hydrogenation of carbon particles covered with a water ice layer in response to $\mathrm{H}$ atom exposure have been studied (Mennella 2008).

Hydrogen-free carbon grain samples with a water ice layer have been exposed at $12 \mathrm{~K}$ to an atomic hydrogen beam obtained by microwave - excited discharge of molecular hydrogen. $\mathrm{H}$ atoms were cooled to a temperature of $80 \mathrm{~K}$. The effects of $\mathrm{H}$ atom irradiation were studied with IR spectroscopy. Figure 1 shows the results obtained for carbon grains

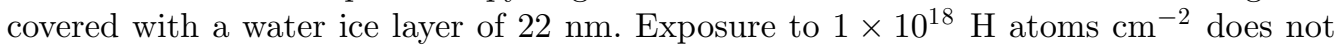
activate the aliphatic and aromatic $\mathrm{C}-\mathrm{H}$ bonds, while, quite surprisingly, the activation of a band at $2883 \mathrm{~cm}^{-1}(3.47 \mu \mathrm{m})$ is observed. In a control experiment, $\mathrm{H}$ irradiation of a water ice layer deposited on a CsI substrate, shows that no feature appears in the $\mathrm{C}-\mathrm{H}$ stretching region (see Figure 1), indicating that the absorption at $3.47 \mu \mathrm{m}$ is activated in carbon grains by $\mathrm{H}$ atom exposure. Following Allamandola et al. (1992), the band at $2883 \mathrm{~cm}^{-1}(3.47 \mu \mathrm{m})$ has been attributed to the $\mathrm{C}-\mathrm{H}$ vibration of tertiary $s p^{3}$ carbon atoms. The $3.47 \mu \mathrm{m}$ band had been identified as a component of the $3.4 \mu \mathrm{m}$ feature of hydrogenated amorphous carbon film using a line shape fitting analysis (Grishko \& Duley 2000).

These experiments have shown that $\mathrm{H}$ irradiation of carbon grains under simulated dense cloud conditions does not activate the formation of aliphatic $\mathrm{C}-\mathrm{H}$ bonds. This result confirms that the assumption about inhibition of formation of this type of bonds in dense clouds is correct. On the other hand, the activation of the $3.47 \mu \mathrm{m}$ band is observed. The different behaviour of the two types of $\mathrm{C}-\mathrm{H}$ bonds has been interpreted in terms of different activation energies for $\mathrm{C}-\mathrm{H}$ bond formation in aliphatic and tertiary $s p^{3}$ carbon sites. $\mathrm{H}$ atoms, impinging on the sample, interact with the water layer at $12 \mathrm{~K}$. Some of the $\mathrm{H}$ atoms are adsorbed in the water ice, while the remainder reach carbon grains. However, due to the interaction with the ice layer, their temperature is lowered well below the activation temperature of $\sim 70 \mathrm{~K}$ for the formation of $\mathrm{C}-\mathrm{H}$ bonds in the $\mathrm{CH}_{2}$ and $\mathrm{CH}_{3}$ groups (Mennella 2006).

On the other hand, the observed formation of $\mathrm{C}-\mathrm{H}$ bonds in tertiary $s p^{3}$ carbon sites implies a negligible activation energy for these bonds.

Figure 2 shows the comparison of the profile of the band activated by $\mathrm{H}$ atoms in carbon grains with a water ice cap with that of interstellar sources. The interstellar spectra (from Brooke et al. 1999) refer to a quiescent region (Elias 16 in the Taurus cloud) and two young stellar objects (GL 2591 and W51). For two massive protostars (GL 989 and GL 2136), Dartois et al. (2002) have derived a profile of the $3.47 \mu \mathrm{m}$ band broader than that obtained by other authors (e.g., Brooke et al. 1999). This result is a consequence of a different choice of the continuum used to obtain the optical depth of the feature (for more details, see Dartois et al. 2002). Note that also in the case of a broader interstellar feature the laboratory band remains compatible with it, accounting for only a part of the overall band profile. The spectral compatibility of an analog material is only a first step towards a solid assignment of the interstellar feature. Formation and destruction cross sections have to be estimated to see whether the proposed carrier is compatible, from the evolutionary point of view, with the conditions present in dense clouds.

The C-H stretching vibration of tertiary $s p^{3}$ carbon atoms, which was proposed as responsible for the $3.47 \mu \mathrm{m}$ band by Allamandola et al. (1992), has been activated in carbon grains through experiments simulating dense cloud conditions. These authors proposed diamond grains as carrier of the feature, a grain population different from the carrier of the diffuse medium $3.4 \mu \mathrm{m}$ band. The laboratory results suggest that there is no need to invoke a separate grain population. As a consequence of evolutionary 


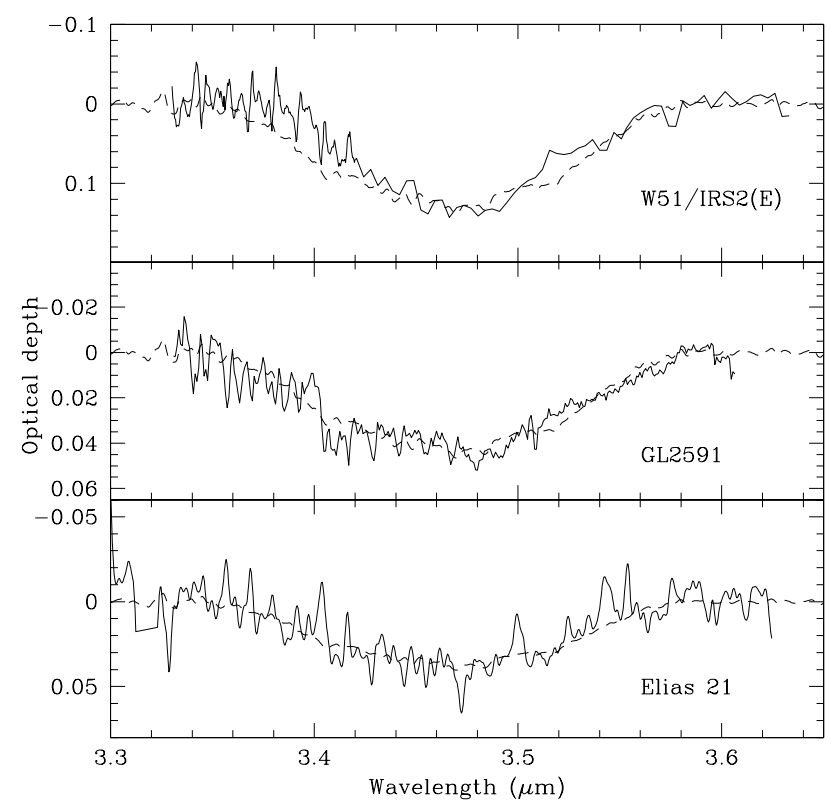

Figure 2. Comparison of the $3.47 \mu \mathrm{m}$ band activated by $\mathrm{H}$ atom exposure in carbon grains covered with a water ice layer (thin dashed lines) with that of three dense clouds (solid lines) (Brooke et al. 1999).

transformations carbon grains responsible for the $3.4 \mu \mathrm{m}$ band in the diffuse ISM can absorb at $3.47 \mu \mathrm{m}$ in dense clouds.

\section{Acknowledgements}

This work has been supported by ASI, INAF and MIUR research contracts.

\section{References}

Allamandola, L. J., Sandford, S. A., Tielens, A. G. G. M., \& Herbst, T. M. 1992, ApJ, 399, 134

Allamandola, L. J., Sandford, S. A., Tielens, A. G. G. M., \& Herbst, T. M. 1993, Science, 260, 64

Brooke, T. Y., Sellgren, K., \& Geballe T. R. 1999, ApJ, 517, 883

Brooke, T. Y., Sellgren, K. \& Smith, R. G. 1996, ApJ, 459, 209

Chiar, J. E., Adamson, A. J. \& Whittet, D. C. B. 1996, ApJ, 472, 665

Dartois, E., d'Hendecourt, L., Thi, W., Pontoppidan, K. M., \& van Dishoeck E. F. 2002, A\& A, 394,1057

Grishko, V. I. \& Duley, W. W. 2000, ApJ (Letter), 543, L85

Mennella, V. 2006, ApJ (Letter), 647, L49

Mennella, V. 2008, ApJ (Letter), submitted

Mennella, V., Baratta, G. A., Esposito, A., Ferini, G., \& Pendleton, Y. J. 2003, ApJ, 587, 727

Mennella, V., Brucato, J. R., Colangeli, L., \& Palumbo, P. 1999, ApJ, 524, L71

Mennella, V., Brucato, J. R., Colangeli, L., \& Palumbo, P. 2002, ApJ, 569, 531

Mennella, V., Muñoz Caro, G., Ruiterkam, R., Schutte, W. A., Greenberg, J. M., Brucato, J. R., \& Colangeli, L. 2001, A\&AA, 367, 355

Muñoz Caro, G., Ruiterkam, R., Schutte, W. A., Greenberg, J. M., \& Mennella, V. 2001, A\&A, 367,347

Pendleton, Y. J., Sandford, S. A., Allamandola, L. J., Tielens, A. G. G. M., \& Sellgren, K. 1994, ApJ, 437, 683

Sandford, S. A., Allamandola, L. J., Tielens, A. G. G. M., Sellgren, K., Tapia, M., \& Pendleton, Y. 1991, ApJ, 371, 607

Sellgren, K., Smith, R. G., \& Brooke, T. Y. 1994, ApJ, 433, 179 


\section{Discussion}

BERNSTEIN: I understand that in your experiments the over layer of water suppresses the formation of the aliphatic C-H stretch at 3.4 microns. However, I would be cautious about strong statements that only the tertiary $\mathrm{C}-\mathrm{H}$ can form in dense clouds. Earlier today we heard from Dr. Cuppen, who talked about $\mathrm{H}$ atom addition to carbon monoxide forming reduce compounds. I also showed earlier that the photolysis of PAHs in water ice, where hydrogen atoms are generated in situ, can add hydrogen atoms to aromatic systems.

Mennella: For my samples, I am evaluated the formation cross-section and there is a small activation energy for the aliphatic component. On the other hand, there is destruction of the aliphatic bonds by UV photons and cosmic rays. I do not see why if you produce the aliphatic bonds by photolysis of ices you do not see aliphatic feature in dense regions. You should explain to me why the aliphatic modes are lacking in dense regions while you can observe the 3.47 micron band. The results of the experiments I have presented indicate a possible origin for the difference between diffuse and dense medium spectral behaviour.

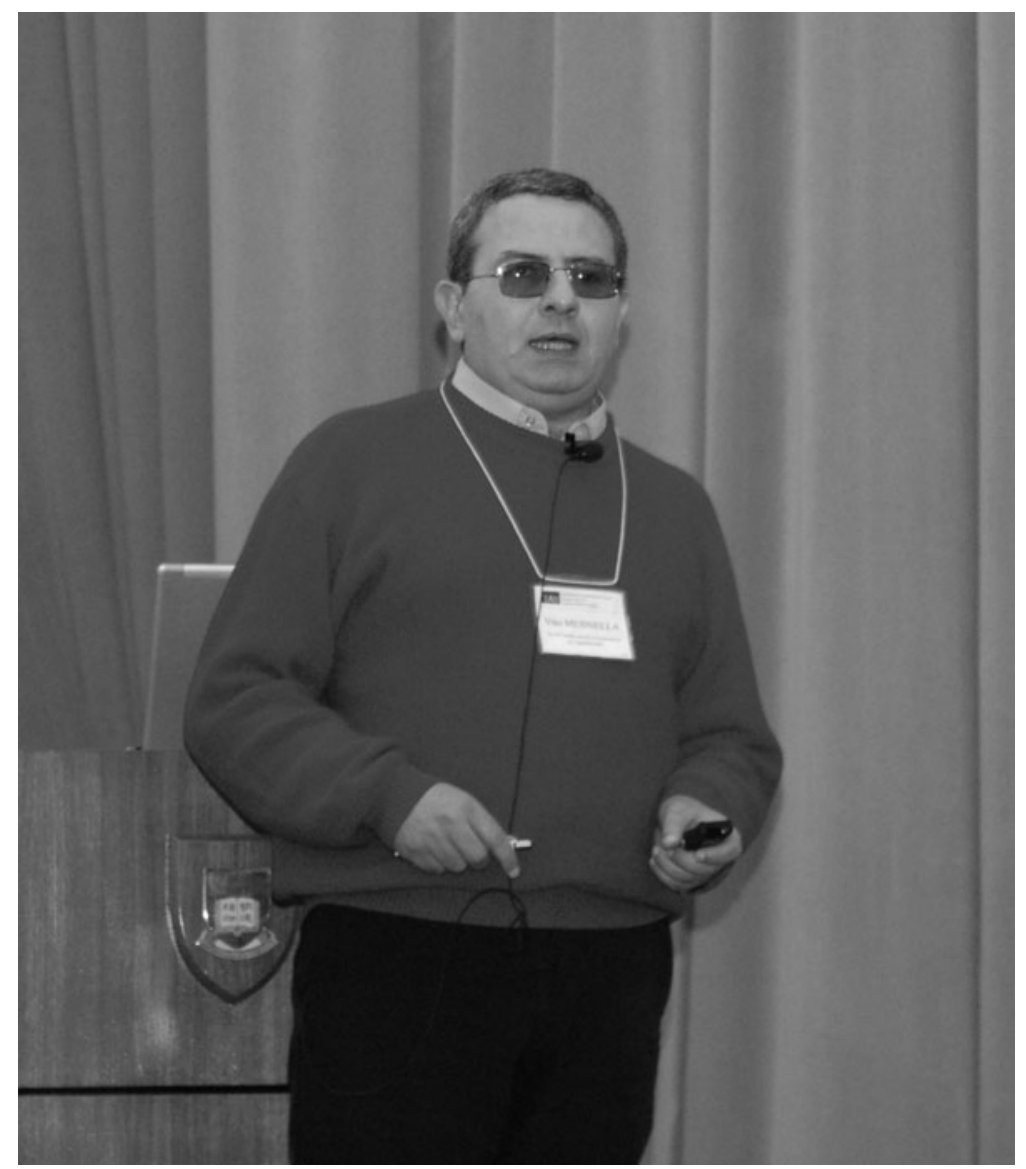

Vito Mennella talking about the evolution of organic material in dense clouds. 


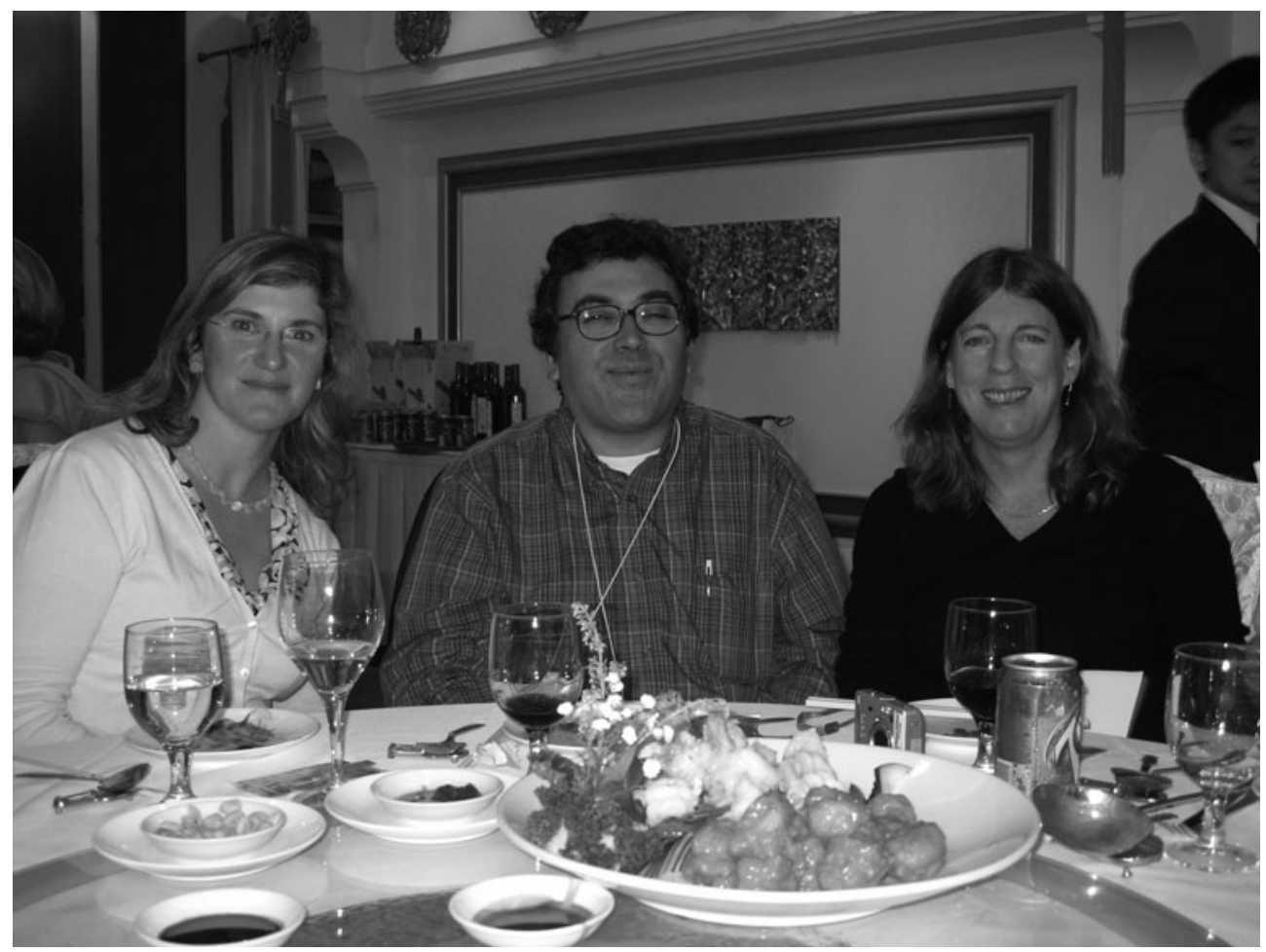

From left to right: Susana Kglesias-Groth, Arturo and Anna Manchado at the banquet. 